\title{
Group “G” B-Hemolytic Streptococcus a New Important Causative Organism of Localised Intra-Abdominal Abscess
}

\section{Shiv Chopra ${ }^{1 *}$, Aneja VK², Raman Sardana ${ }^{2}$ and Leena Mehndiratta ${ }^{2}$}

${ }^{1}$ Department of General and Gastrointestinal Surgery, Indraprastha Apollo Hospital Delhi-Mathura Road, Sarita Vihar, New Delhi-110076, India

${ }^{2}$ Department of Internal Medicine, Indraprastha Apollo Hospital, Delhi-Mathura Road, Sarita Vihar, New Delhi-110076, India

\begin{abstract}
Group "G" Streptococcus is an ubiquitous non-pathogenic commensal organism found in the skin, throat, gut, appendix and the female genital tract. As per our review of the available literature we present the first reported case of localized intra-abdominal abscess caused by Group g streptoccoccus (GGS) also seen first time in our institution which is a rare but an important cause when after the requisite Surgical procedure-drainage and antibiotic regimens the post-operative pyrexia/symptoms do not settle down with rising counts then one should start thinking about upgrading the antibiotics to meropenem+ciprofloxacin/vancomycin/teichoplanin along-with the existing anaerobic cover. In our case blood cultures were negative, only the pus culture-liquid subcultures grew large colony gram positive cocci on the $1^{\text {st }}$ postoperative day with further identification as Group G Streptococcus (GGS) with the sensitivity pattern on the $3^{\text {rd }}$ postoperative day identified the organism as large-so a very good microbiology department team is necessary which in our case was instrumental in the management of our patient with a very high index of suspicion. This case is being reported for the reason of its rarity. Especially reported in patients who are diabetics/malignancies/respiratory infections/immunosuppressed/chronic leg ulcers/pet dogs at home it is gaining importance as an organism for causing deep-seated abscesses in the body especially the abdomen which are usually multiple or as peritonitis. Another important reason mentioned in literature for immediate and urgent drainage of abscess caused by GGS infections is that this may lead to a very serious streptococcal toxic shock syndrome having a very fulminant and rapid downhill course with GGS bacteremia having a high 30 days mortality rate of to $15 \%$.
\end{abstract}

Keywords: Streptococcus; Non-pathogenic; Meropenem; Diabetic

\section{Case Report}

58 years lady, borderline diabetic presented to us in the emergency on oral hypoglycemic/ antihypertensive therapy, with a 6 days history of abdominal pain right side abdomen and right iliac fossa which markedly increased over last 36 hours. She had 2 episodes of chills with high grade fever 102 degrees fahrenheit, with raised counts of up-to $14,500 / \mathrm{mm}^{3}$. Her ultrasound examination done a day back showed thickened inflamed bowel wall-appendix involved in the ileocaecal region without any collection, rest of the viscera was normal. On examination we saw a moderately dehydrated lady, fully conscious, anxious, febrile 99 degree $\mathrm{F}$ temperature, pulse 90/minute, blood pressure-120/70, respiratory rate of $18 /$ minute, abdominal findings revealed infra-umbilical midline LSCS scar (28 years ago), severe tenderness right iliac fossa, with a rebound tenderness present, PR examination showed fecal matter present. Her labs revealed raised counts $12,400 / \mathrm{mm}^{3}$, neurtophils of $86 \%$, amylase/lipase within normal limits, rest of her labs were essentially normal. After urgent Preanaesthetic checkup/Full informed high risk consent she was taken up for surgery. Diagnostic laparoscopy revealed lot of adhesions with purulent exudate in the right paracolic gutter, with tight confines so it was decided to convert to open laparotomy by entering the abdomen via the old scar in the midline and entering the peritoneal cavity onto right side of the adhesions. The omentum was densely adherent to anterior abdominal wall in the midline effectively dividing the lower abdomen into right and left halves. The ileocaecal region was identified and the mesentry of the terminal ileum, caecum was grossly edematous and thickened, almost about 10 to $12 \mathrm{~cm}$ thickness and on lifting the cecum in the retro-caecal area a very small collection was drained only about $10 \mathrm{ml}$ thick very foul smelling yellow pus which was removed in a syringe and sent for culture/sensitivity, appendix was not seen, probably liquefied only the base was seen identified by the converging taenia on the caecum, which was ligated, its thickened inflamed mesentery was removed and sent for histopathological examination, the rest of the intestine appeared viable but friable so too much handling was also avoided, the uterus/appendages and rest of viscera on examination appeared normal, peritoneal toileting was done with copious warm saline and a thick bore tube drain 32 french was left in the pelvis, the left half of the abdomen did not appear to be contaminated so it was now decided to close the abdomen with interrupted prolene 1-0 figure of " 8 " sutures, in layers. She had been started on cefoperazone+sulbactam/ metronidazole/amikacin injections, and shifted to the icu. On $1^{\text {st }}$ postoperative day her counts rose to $14,700 / \mathrm{mm}$, neutrophils of $89 \%$, Ultrasound examination abdomen-did not reveal any collection/repeat chest X-ray was normal. On the $2^{\text {nd }}$ postoperative day she showed a febrile peak of 99 degree $F$, so it was decided by the team to upgrade her antibiotics to inj meropenem+inj ciprofloxacin (on post-op day 2) +inj teichoplanin (on post-op day 3), and stopping inj cefoperazonesulbactam (pot-op day 2) /amikacin (post-op day 3) and continuing with metronidazole. The microbiology dept alerted us to the growth of Gram positive cocci on liquid subcultures on the 1st post-operative day and gave us the sensitivities on the $3^{\text {rd }}$ post-operative day for the GGS. Her counts/pyrexia settled within 48 hours ( $4^{\text {th }}$ post-operative day) of the changed regimen. She remained afebrile from $5^{\text {th }}$ postoperative day onwards, her sputum cultures showed alpha-haemolytic streptoccus and budding yeast cells-as contaminants from oral flora, urine (48

*Corresponding author: Shiv Chopra, Department of General and Gastrointestinal Surgery, Indraprastha Apollo Hospital, Delhi-Mathura Road, Sarita Vihar, New Delhi-110076, India, Tel: 0112692 5858; E-mail: sbchopra2004@yahoo.com, drshivchopra@apollohospitalsdelhi.com

Received December 25, 2013; Accepted February 12, 2014; Published February 14,2014

Citation: Chopra S, Aneja VK, Sardana R, Mehndiratta L (2014) Group "G" B-Hemolytic Streptococcus a New Important Causative Organism of Localised Intra-Abdominal Abscess. J Clin Case Rep 4: 340. doi:10.4172/2165-7920.1000340

Copyright: @ 2014 Chopra S, et al. This is an open-access article distributed under the terms of the Creative Commons Attribution License, which permits unrestricted use, distribution, and reproduction in any medium, provided the original author and source are credited. 
hrs)/blood cultures (1 week) cultures did not show any growth and she was discharged home on the 10th post op day with normal counts of $9,300 / \mathrm{cmm}^{3}$, neutophils $60 \%$, in a fully recovered condition with further antibiotics of augmentin (amoxycillin+clavulanic acid) for 10 days. On retrospective history she had no pets at home, but she recalled having had vague abdominal discomfort since last 1 month, also some sore throat issues about 10 days back which resolved on mouth gargles only within 3 days, which she did not consider important and she had no leg ulcers. She was discharged without any antihypertensives/oral hypoglycemic drugs and only dietary advice (Figure 1).

\section{Discussion}

This is the first reported case from our institution of large colony B-hemolytic Group G streptococcus as a cause of intra-abdominal abscess probably a spontaneous case, and also the first reported case in the world which was small and localised. They may be associated with active respiratory infections also [1-3] of which $6 \%$ cases maybe lower respiratory tract infection [3] this was not so in our case as only alpha hemolytic streptococci as contaminants were seen on sputum cultures. They may be poly-microbial infections like staphylococcus aureus, gram negative or anaerobic organisms upto $41 \%$ of cases [4-6] and usually only one bacterium may be cultured $[1,4]$ as was seen in our case. The case reported by [1] was a female patient diabetic 52 years presenting with long course of illness of about 4 weeks with abdominal pain and tenderness of few weeks history, abdominal lump left upper quadrant but had multiple involvement with abscesses of abdominal wall/omentum and abdominal cavity detected on computerized axial tomography scan of the abdomen with a history of alcohol abuse, mildly raised counts $11,600 / \mathrm{mm}^{3}$, negative blood cultures, history of 25 kilogram weight loss in 4 weeks and a history of a visit to her general practitioner for pharyngitis one month ago. Our patient was also a known diabetic/hypertensive/obese, 96 kilograms, but had a shorter more acute history of 6 days only with raised counts of 14,500/ $\mathrm{mm} 3$, fever with chills and rigors, although on retrospective history she did give history of vague abdominal discomfort since last 1 month. There was no history of weight loss, or pharyngitis or cellulitis/skin infections/malignancies as was reported by other workers $[4,5]$ and also had no pets especially dogs and no leg ulcers [4]. She had no history of alcohol abuse, pure vegetarian. Her blood cultures were also negative after 1 week incubation as seen in the [1] however extra abdominal

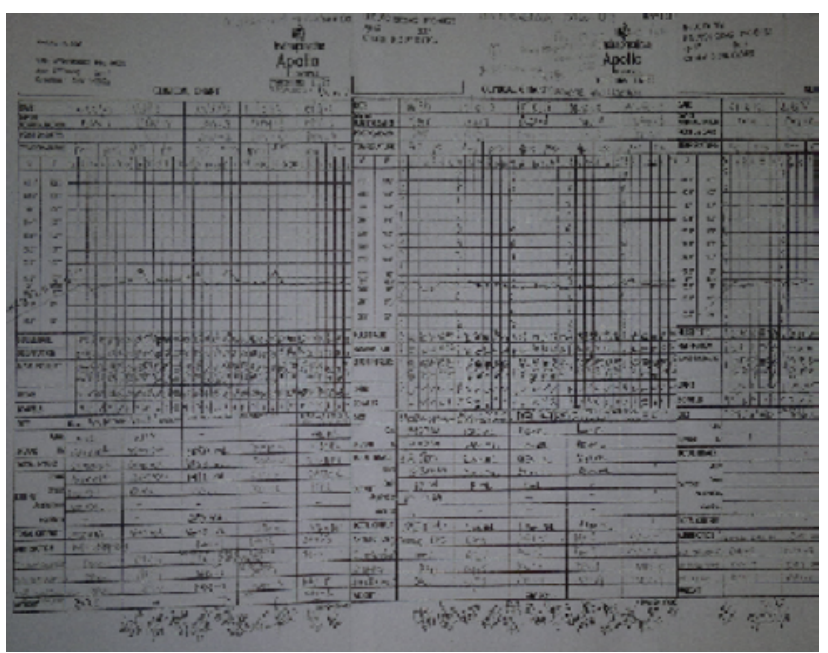

Figure 1: Nursing chart showing the post-operative low grade pyrexia $2^{\text {nd }}$ to $4^{\text {th }}$ post-operative days. infections by GGS had positive blood cultures as seen by other workers [2,4-6] as high as $41 \%(128 / 314$ cases) as reported by [6]. A history of skin infections/disruption of skin barrier [2-6] was seen in twothirds cases of GGS bacteremias 53\% being second only to Group A streptococcus 58\% infections [6] Group C streptococcus Large colony generally include several species like S. Dysgalactae, S. equisimilis, S. zooepidemicus and S. equi [7]. Average length of stay for all diabetic patients with GGS infections all over the body was longer (14.6) days than those who were not having diabetes mellitus [6]. DeBrahandre's case was discharged after 17 days [1] which although was drained laparoscopically while our case underwent emergency diagnostic laparoscopy and converted to open exploratory laparotomy with a prompt follow up and up-gradation of antibiotics in response to further liquid subcultures and our case was discharged on post-operative day 10. GGS have a low virulence and we should not underestimate the pathogenic potential of this so common a ubiquitous organism [2] which may require very specific antibiotics and we should not ignore the low grade peaks which may continue as seen in our case despite a very good antibiotic combination of third generation cephalosporin/ aminoglycoside/metronidazole which are usually very successful in controlling most of the infections in our routine practice. In our case it was only on liquid subcultures that on the $3^{\text {rd }}$ day a growth of gram positive cocci were seen and a close discussion and co-ordination with the microbiologist and physician resulted in the successful treatment of this patient. Per-operatively the mesentry of the terminal 6-7 inches of ileum and the retroperitoneal attachments of caecum and proximal ascending colon were found to be grossly inflamed, edematous and thickened, $10-12 \mathrm{~cm}$ thick in contrast to the mesentry of the rest of small intestine and the rest of the abdomen which was cut off by the adhesions of previous surgery, thus effectively localising this severe infection to the right lower quadrant of abdomen, which was probably good for this patient. The severity of the infection can be gauged by the finding of the hugely edematous mesentry of terminal ileum which was totally out of proportion to the very small quantity about $10 \mathrm{ml}$ of the evacuated pus and the appendix which was also conspicous by its absence as it had liquefied, leaving only the base and mesentry of appendix which on being sent for histopathological examination revealed fat necrosis, active granulation tissue and severe acute inflammatory exudate in the fibrofatty tissue. Another thing is that the routine 48 hours culture trend as done in smaller centres would have missed this bacterium altogether. It is reported in literature that there is an increasing incidence of GGS infections either due to increased reporting, vigilant microbiologists or increasing virulence $[3,4,6]$. Whether this is representing an emerging new trend of pathogenicity in an apparently non-pathogenic organism where the pet is now biting the hand of its master! Further work, studies and close co-operation of clinicians with the microbiologists needs to be done. Also many microbiologists only classify the Streptococci as only Group A,B,G and $\mathrm{F}$, finding earlier specification quite superfluous.

\section{References}

1. De Brabandere K, Vanpaemel G, Verheyen $L$ (2008) Spontaneous abscesses of the abdominal wall, omentum and abdominal cavity caused by group $G$ streptococci: a case report. Acta Chir Belg 108: 765-767.

2. Lestin F, Mann S, Podbielski A (2008) Spondylodiscitis and paraspinal abscess caused by beta-haemolytic group $\mathrm{G}$ streptococci spreading from infected leg ulcers. J Med Microbiol 57: 1157-1160.

3. Wong SS, Lin YS, Mathew L, Rajagopal L, Sepkowitz D (2009) Increase in group $G$ streptococcal infections in a community hospital, New York, USA Emerg Infect Dis 15: 991-993.

4. Lam MM, Clarridge JE 3rd, Young EJ, Mizuki S (2007) The other group G 
Citation: Chopra S, Aneja VK, Sardana R, Mehndiratta L (2014) Group "G" B-Hemolytic Streptococcus a New Important Causative Organism of Localised Intra-Abdominal Abscess. J Clin Case Rep 4: 340. doi:10.4172/2165-7920.1000340

Streptococcus: increased detection of Streptococcus canis ulcer infections in dog owners. J Clin Microbiol 45: 2327-2329.

5. Auckenthaler R, Hermans PE, Washington JA 2nd (1983) Group G streptococcal bacteremia: clinical study and review of the literature. Rev Infect Dis 5: 196-204.
6. Rantala S, Vuopio-Varkila J, Vuento R, Huhtala H, Syrjänen J (2009) Clinical presentations and epidemiology of beta-haemolytic streptococcal bacteraemia: a population-based study. Clin Microbiol Infect 15: 286-288.

7. Jhonson CC, TunkelAR. Viridans streptococci and groups C and G Streptococci, and Gemella Species. 